\title{
A PÓS GRADUAÇÃO EM ENFERMAGEM NA REGIÃO NORTE: Situação atual e perspectivas.
}

\author{
Maria do Socorro Batista de Sousa*
}

RESUMO - Trata da pós graduação em enfermagem na Região Norte, com o objetivo de levantar $\Theta$ analisar a situação atual da pós graduação em enfermagem nas Instituições de Ensino Superior da Amazônia Legal, que possuem o curso de enfermagem.

ABSTRACT - It is about pos graduation in nursing, in Região Norte, with the finality of getting records and analize the actual situation of pos graduation in Nursing in Institutions of superior teaching of Amazonia legal, who have this nursing course.

\section{INTRODUÇĀO}

Para que se obtenha novo padrão tecnológico, adaptado ao Desenvolvimento Regional, é fundamental que se amplie e aprofunde o conhecimento na busca de novas soluções e oportunidades. Portanto, a adoção de formulações alternativas para encaminhamento dos problemas regionais, torna-se necessária a fim de que as Universidades, como centro de produção do saber, sejam as pioneiras, inovadoras, com o objetivo de aprimorar o estudante, o docente e/ou assistente, permitindo alcançar elevado padrão de competência técnico-profissional e científica que são exigidos pelas condições de pesquisas e necessidade de treinamento avançado, não facilmente adquirido no âmbito da graduação.

Entretanto, a pós graduação é importante componente do Sistema da Ciência e Tecnologia, sob o aspecto de qualificação docente, a qual constitui ponto de fortalecimento e ampliação da informação científica.

Diante dessa realidade, o Protocolo de Integração das Universidades Federais da Amazônia Legal, denominado Protocolo de Integração, criado em 1979 pelos Reitores das referidas Instituições de Ensino Superior, composto pelos estados do Acre, Amazonas, Mato Grosso, Maranhão, Pará e Rondônia, com a finalidade de consolidar um programa de racionalização de condições e de colaboração recíproca, como experimento útil para abordagem conjuntural de problemas comuns, ações, programas de trabalho e principios de uma política comprometida com a urgente qualidade do atual nivel de ensino, reune-se para elaborar, entre outros, um documento conjunto visando o Programa de Pós Graduação da Área Norte, tratando, especialmente na área da saúde, do curso de enfermagem. A integração dessas Universidades tornar-se-á vital para a região pois viabilizará programas de qualificação dos recursos humanos até então não implantados e nem priorizados, em especial na área de saúde.
A Amazônia Legal, com seus cinco milhōes de quilômetros quadrados, aproximadamente $60 \%$ do território brasileiro, caracteriza-se por apresentar problemas específicos, tomando-se imprescindível a adoção de medidas exclusivas na solução de problemas. Somente com a utilização de pesquisas conseguir-se-á a transformação efetiva do desnivel em nossa região quando comparadas as atuais condições, a despeito do distanciamento geográfico existente, da mesma aos grandes centros de pos graduação, tendo em vista a carência de infra estrutura no campo da saúde, abrangendo todos os cursos, onde destacamos o de enfermagem. cuja urgência de desenvolvimento, julgamos prioritária.

A assistência à saúde solicita do enfermeiro conhecimentos de natureza especiallizada, os quais passam a influenciar o atendimento e a atividade, desde a utilização de tarefas de rotina até procedimentos que variam em dificuldades e complexidade demandando alto grau de experiência técnico-científica e conhecimentos teóricos nos diversos ramos da ciência.. Esse enriquecimento profissional promove mutações significativas no campo social, fortalecendo o preparo de enfermeiros para o exercŕcio de sua cidadania, no papel de educador e orientador. Para isso, tornam-se necessários estímulos e medidas, como uma política de educação mais voltada para a problemática dessa sub-área.

A formação de pós graduação do enfermeiro quer a nível de "stricto sensu" quer a nível de "lato sensu" deve ser visto, segundo RHODUS (1977), em relação ao desenvolvimento científico dos valores sociais vigentes e fatores que originam mudanças e influenciam o ensino de $3{ }^{\circ}$ grau e o sistema de prestação de assistência à saúde da população.

O objetivo primordial proposto é levantar e analisar a situação atual da pós graduação em enfermagem nas Universidades da Amazônia Legal, suas condiçōes atuais e perspectivas como contribuição a futuros estudos nessa área.

\footnotetext{
* Professor Adjunto do Departamento de Enfermagem do Centro Ciências da Saúde. Mestre em Enfermagem.
} 


\section{REVISÃO DA LITERATURA}

RHODUS (1977) comentando fatos sobre a pos graduação salienta, no estudo realizado, o curso de mestrado da Escola de Enfermagem Ana Neri, da Universidade Federal do Rio de Janeiro - UFRJ. onde apresenta um retrospecto do desenvolvimento da pós graduação em enfermagem a partir do Sistema Nightingale, mostrando e analisando a situação da mesma, naquela Universidade, evidenciando solicitaçōes e tendências que norteiam o papel do enfermeiro como elemento da equipe de saúde, lider da educação à serviço da enfermagem.

RHODUS (1977) evidencia, também, em seu estudo perspectivas de enfermagem estabelecendo nítida distinção entre situações enfrentadas pelo profissional "em termos de atender a dois propósitos, até certo ponto irreconciliáveis: servir o hospital e atender o objetivo do ensino". Destaca ainda a autora um aspecto mais particular, quando trata das indicações para a enfermagem ensino-serviço e que o ensino da enfermagem prevê as atividades dos alunos na instituições hospitalares e na comunidade, em seqüência ao ensino formal, teórico, ministrado em salas de aulas, fazendo-se necessário que os campos de estágio of ereçam condições para desenvolver tal ensino, seja a nível de graduação e/ou pós graduação.

Reconhece, ainda, RHODUS (1977) que a pesquisa nessa profissão tem recebido pouca atenção dos estudiosos, causando um crescimento lento em conseqüência de recursos limitados e dos reduzidos resultados alcançados.

PELÁ et alii (1982) fazem uma análise da "situação atual dos cursos e produção científica da pós graduação da Escola de Enfermagem de Ribeirãc Preto, abrangendo o período de 1978 a 1980'. Esclarecem que para o desenvolvimento do corpo docente e o aperf eiçoamento do ensino e da pesquisa, houve necessidade da participação de uma equipe de docentes dessa Escola e de outros centros de pós graduação. Referem ser fundamentais a colaboração de outros departamento. Mencionam, ainda, o início do curso de Mestrado em Enfermagem, visando atender inicialmente a própria demanda da escola, não levando em consideração as áreas de atuação docente, por ser o momento crítico. Realizaram estudos avançados dando ênfase aos aspectos humanos da enfermagem e ao desenvolvimento da pesquisa nessa árera, com enfoque na prevenção primária em enfermagem psiquiátrica, especialmente, inserindo-a em todas as especialidades.

A enfermagem como profissão emergente, ainda nos nossos dias, prepara-se para consolidar sua tradição em pesquisa, o que nos propicia uma reavaliação dos aspectos proeminentes da área de saúde, motivando-nos à constatação de que as deficiências da enfermagem podem converter-se em habilidades, correspondendo ao que se deseja, no que diz respeito à saúde do indivíduo e da sociedade, se acompanhados de base científica para a prática.

GONÇALVES (1982) relata o empenho para a idealização do "Projeto de Pós Graduação em Enfermagem Stricto Sensu na Região Sul", juntamente com a "implantação do curso de Mestrado em Ciência da Enfermagem opção a saúde do adulto, na Universidade Federal de Santa Catarina, em 1976". Relata que a essência do "espírito do projeto se fundamentava na cooperação inter instittucional visando ao aproveitamento racional dos recursos didáticos, humanos e materiais bem como facilitar o acesso dos docentes das proprias universidades aos cursos evidenciando a contribuição da melhoria do ensino graças à capacitação docente no Departamento de Enfermagem". Ressalta resultados positivos do curso de especialização realizado em caráter permanente e em relação à produção científica na formação de grupos emergentes engajados na pesquisa apesar de não estarem claramente definidas as linhas das mesmas.

BASSALO et alii (1984) apresentam documento sobre a situação da pesquisa e da pós graduaçãao na Universidade Federal do Pará. Relatam a situação do Centro de Ciências da Saúde sugerindo estratégias para a definição de linhas de pesquisa e providências necessárias relativas às perspectivas realizadas pelos docentes e discentes no Centro de Ciências da Saúde, como em toda a Universidade Federal do Pará". Mencionam que, exceto a Residência médica em pediatria, não existe, em caráter permanente, na UFPa., cursos de especialização "lato sensu" em nível de especialização, na área da saúde. Destacam a hipótese da UFPa. ser "o centro polatizador do desenvolvimento da Amazônia, possibilitando através desses cursos, o estudo e a pesquisa nesta área, daŕ a urgente necessidade de criação e implantação permanente dos mesmos".

O documento básico da UNIVERSIDADE FEDERAL DO ACRE (1986) coloca em evidência a imperiosa tarefa de reestudar e reelaborar o conhecimento acumulado de outras regiōes os quais não são prontamente aplicados para responder às suas necessidades. Aspira por "um corpo de conhecimentos centrados nessa região" que necessite de uma definição política que leve em consideração a necessidade de um esforço comum, centrado no estudo de seus problemas mais especificos, com vista a determinar soluções técnicas adequadas ao desenvolvimento regional, para integrar-se ao país como um todo". Comenta que a "Região Amazônica já dispōe de centros de estudos, Universidades, Instituto de Pesquisas, que podem ser aparelhados adequadamente para transformarem-se em órgãos de produção de conhecimentos para soluções técnicas adequados em situações locais".

O documento da UNIVERSIDADE FEDERAL DO ACRE (1986) não menciona programas especi- 
ficos da área de saúde, nem, em especial, da enfermagem, entretanto menciona interesse quanto a "necessidade de fomentar a pós graduação como um dos mecanismos para a formação de recursos humanos de alto nível".

A FUNDAÇÃO DA UNIVERSIDADE DE MATO GROSSO (1986) através de seu documento básico informa sobre o ingresso dessa Universidade no Programa PICD/CAPES, a partir de 1975 o que fez crescer o número de docentes titulados de 2 mestres em 1974 para 224 mestres e 20 doutores, em 1986. Relata o apoio organização e manutenção da Universidade aos núcleos de pesquisa e pós graduação sem contudo referir-se a área da saúde, apesar de estar esta ordenada entre as prioridades, não fazendo qualquer referência à enfermagem.

O documento básico da UNIVERSIDADE FEDERAL DO PARÁ (1986) trata entre outras abordagens da pós graduação e pesquisa diagnosticando os cursos "stricto sensu" e "lato sensu" em todas as áreas, incluindo a saúde. Diz o documento que a UFPa. não pode estar somente voltada para o ensino acadêmico convencional, alegando que lhe é fundamental a preparação da mão de obra habilitada para o mercado de trabalho existente, embora muitas áreas este jam bastante inflacionadas. Apresenta projetos, priorizando-os. Entre estes projetos estão inclúfdo os da área da saúde. Relaciona os cursos de mestrado e doutorado existentes na UFPa. dos quais, nenhum na área da saúde. Dos 93 (noventa e três) cursos de especialização e aperfeiçoamento apenas 5\% encontram-se na área da saúde.

MACHADO (1986) refere a participaçãoa da Região Norte no Sistema Nacional de Pós Graduação, "com 10 programas de pós graduação "stricto sensu", reprentando 10 cursos de mestrados e 5 de doutorado, o que representa $1 \%$ dos cursos de mestrado e $1 \%$ dos cursos de doutorado existentes no Pais". Desses programas nenhum deles encontra-se vinculado a área da saúde.

$O$ documento da Fundação Universidade do Amazonas e Instituto Nacional de Pesquisas da Amazônia (1986) destacam os cursos de pós graduação do convênio INPA/FUA, cu jo programa teve início em 1973, e vem crescendo até hoje. Cita as áres priorizadas dentro do Programa de Pós Graduação INPA/FUA salientando a prioridade dada à área da saúde, particularmente à sub-área nutrição que desde 1984 realiza curso em nível de mestrado.

\section{METODOLOGIA}

Tomamos para estudo a Escola de Enfermagem da Universidade Federal do Acre, a Escola de Enfermagem da Universidade Federal do Maranhão, a Escola de Enfermagem da Universidade Federal do Mato Grosso, a Escola de Enfermagem da Universidade Federal do Pará, a Escola de Enfermagem "Magalhães Barata", no Pará, e à Escola de Enfer- magem de Manaus.

\section{TÉCNICAS}

\section{1 - Questionários}

Foram coletados dados através de questionários, encaminhados por carta-ofício, à cada instituição selecionada.

\section{2 - Tabelamento Estatístico}

O dados foram tabulados descritivamente com indicação de freqüência e cálculo de percentagem.

\section{RESULTADOS E DISCUSSÓES \\ 5.1 - A Situação Atual da Pós Graduação em Enfermagem na Universidade Federal do Acre.}

O Curso de Graduação em Enfermagem é o único, na área da saúde, na Universidade Federal do Acre, vinculado ao Departamento de Ciências da Saúde.

Teve início em 1986 propondo-se a formar o enfermeiro generalista. Não possui habilitações.

Com o objetivo de contribuir para elevar a qualidade do ensino e assistência ofereceu aos docentes enfermeiros e profissionais da assistência um curso em 1980 sobre Recursos Humanos, na área da saúde, utilizando-se do sistema de módulos, o qual era oferecido durante o período de recesso escolar. Nos meses de julho e agosto de 1980 foi realizado o 1 . módulo, em 1981, nos meses de janeiro a fevereiro, o $2^{\circ}$ módulo sendo o $3^{\circ}$ modulo of erecido nos meses de janeiro e fevereiro de 1982 . Facultando aos participantes a opção de realização dos mesmos, quem concluiu apenas 1 módulo recebeu certificado de extensão e aqueles que completaram os 3 módulos receberam certificado do aperfeiçoamento. Esse curso ocorreu através do convênio firmado entre a Universidade Federal do Acre, Universidade Federal de Pernambuco e SUBIN. De acordo com o número de créditos era diferenciada sua carga horário: para enfermagem 570 horas/ aula com 25 créditos e para ciências, 390 horas/aula com 23 créditos.

No perfodo de 1985 a 1986 a Universidade Federal do Acre of ereceu um Curso de Especialização em Tópicos de Enfermagem Geral, com o objetivo de contribuir na melhoria da assistência de enfermagem ao individuo, familia e comunidades no processo saúde-doença, com carga horária de 450 horas e 30 créditos. Inscreveram-se 28 candidatos tendo concluído apenas 21. Este curso foi realizado através do convênio firmado entre UFAC/MEC/BIDIII.

O Curso de Graduação em Enfermagem através de seu Departamento de Ciências da Saúde nāo oferece cursos de pós graduação, regularmente. Portanto, não possui estrutura e pessoal.

Dos 25 docentes enfermeiros da Universidade 
Federal do Acre, nenhum possui título de Doutor. Apenas 4 possuem Curso de Mestrado sendo, 2 da categoria de prof essor assistente e 2 da categoria de adjunto, representando $16 \%$ dos docentes. Com nivel de espocialização encontram-se 18 professores, sendo 8 auxiliares de ensino e 10 assistentes, correspondendo a $72 . \%$

Em relação ao regime de trabalho 3 docentes, com especialização encontram-se em regime de 20 horas, 10 professores, sendo 2 mestres e 8 especialistas exercem atividades em regime de 40 horas e 9, sendo 2 mestres e 7 especialistas, encontram-se em regime de dedicação exclusiva.

Constatamos, assim que dos 25 docentes da Universidade Federal do Acre, 22 possuem cursos de pós graduação, o que corresponde a $88 \%$ de pessoal com pos graduação, notamos, entretanto, que desses a grande maioria, representada por 12 docentes, ou seja $54,6 \%$ exerce apenas a docência enquanto que 3 realizam pesquisa e docência. Os demais realizam outras atividades, sendo que um deles encontra-se à disposição de outra Instituição.

\section{2 - A Situaçăo Atual da Pós Graduaçăo em Enfermagem na Universidade Federal de Mato Grosso.}

O Departamento de Enfermagem da Universidade Federal de Mato Grosso foi criado em 1976 visando suprir as necessidades regionais de enfermagem, de acordo com as metas do II Plano Nacional de Desenvolvimento (IIPND), com o objetivo de formar o maior número de enfermeiro, no mais curto espaço de tempo. Encontra-se vinculado ao Centro de Ciências Biológicas e da Saúde.

A graduação em enfermagem é oferecida de forma integrada, sem habilitações. É concedido, também, pelo Departamento, o Curso de Licenciatura em Enfermagem.

O pessoal docente foi contratado apos as primeiras turmas de graduação.

As atividades assistenciais são exercidas no hospital universitário e no centro de saúde escola; os enfermeiros admitidos no hospital universitário e centro de saúde escola são contrados como docentes lotados no Departamento de Enfermagem.

O Departamento de Enfermagem envidou esforços para capacitar, a nível de especialização, o maior número de docentes encaminhando-os aos mais diversos cursos do País. Paralelo a esse esforço foi iniciada a capacitação a nível de mestrado. Com o retorno desses docentes será dado início à pós graduação "lato sensu", de maneira mais efetiva e regular.

Em 1983 o Departamento of ereceu um Curso de Especialização em Metodologia de Pesquisa na área da saúde, tendo capacitado 19 docentes enfermeiros.

Em termos de perspectiva está planejado um Curso de Metodologia da Assistência e Administração de Enfermagem, previsto para capacitar 20 docentes enfermeiro e 10 enfermeiros da comunidade.

A Universidade Federal do Mato Grosso possui 58 docentes enfermeiros, dos quais 45 possuem cursos de pós graduação (6 mestres e 39 especialistas) correspondendo a $76 \%$.

\section{3 - A Situação Atual da Pós Graduação em Enfermagem na Universidade Federal do Pará.}

A Universidade Federal do Pará possui um Centro de Ciências da Saúde do qual, entre outros, fazem parte o Departamento de Enfermagem e o Curso de Enfermagem.

O curso de enfermagem foi instalado em 1976 propondo-se a formar enfermeiros com habilitações em Enfermagem de Saúde Pública, Enfermagem Médico-Cinúrgica e Enfermagem Obstétrica, sendo que esta, até o momento, não foi implantada, apesar de constrar da resolução que criou o Curso de Enfermagem.

Através do Centro de Educação da UFPa. é oferecido o Curso de Licenciatura em Enfermagem, restrito a alunos do Curso de Enfermagem da UFPa.

No período de dezembro de 1980 a fevereiro de 1981, durante o recesso escolar, foi realizado o Curso de Metodologia do Ensino e Assistência de Enfermagem, com o objetivo de qualificar e aprimorar os conhecimentos docentes, tendo em vista as dificuldades de deslocamento dos mesmos para outras regiōes. Referido curso foi realizado atraves do Convênio UFPA/CAPES $\mathrm{e}$ assessoramento da UFCe/UFRJ; com carga horária de 480 horas. Foram oferecidas 30 vagas. Inscreveram-se 30 candidatos tendo concluído apenas 23, os quais receberam o titulo de especialistas.

Para 1984 foi programado o Curso de Didática Aplicada à Enfermeagem como forma de possível reorganização do conteúdo curricular e aprofundamento do docente às técnicas de avaliação de desempenho e aprimoramento na tecnologia educacional. Até o momento não foi realizado por falta de recursos financeiros, além das barreiras tecnocrátas.

As dificuldades para realização de novos cursos de especialização são inúmeras.

Dentro da política de capacitação de recursos humanos os docentes são encaminhados para realização de cursos à outras IES. Desde 1977 o Departamento de Enfermagem encaminhou apenas 1 docente para cursar mestrado.

Em termos de perspectivas sente-se necessidade de cursos de pós graduação "lato sensu", não em caráter temporário mas permanente, a fim de atualizar o saber dos docentes e enfermeiros, de acordo com o desenvolvimento da ciência, a fim de que prestem uma assistência de qualidade à comunidade, por considerarmos seus serviços relevantes diante do contexto político-social. 
Aspira, também, o Departamento de Enfermagem, a realização de um curso a nível de "stricto sensu", através de convênios, já existentes em outras regiōes, com financiamento da CEF/FAS/BID/ MEC/CEDATE.

Em relação à pesquica o caminhar tem sido muito lento, no curso de enfermagem da Universidade Federal do Pará. A impossibilidade de contratação de pessoal docente imposta pelo Governo Federal e o número reduzido de docentes, aquém da carga horária semanal oferecida implica a que a grande maioria dos docentes seja lotada apenas em atividades docentes impedindo-os de realizar atividades de pesquisa ou extensão, com alocação de carga horária.

Os trabalhos de pesquisa dos docentes, quando realizados, são por iniciativa pessoal, sem qualquer ônus para a Instituiçăo.

A Universidade Federal do Pará possui no Departamento de Enfermagem 26 docentes enfermeiros. Destes nenhum possui curso de Doutorado. do total, 5 são mestres e 18 especialistas. Dos mestres 1 é professor assistente e 4 são professores adjuntos. Os especialistas são 4 auxiliares de ensino e 14 assistentes.

Verificamos, assim que dos 26 docentes enfermeiros 22 são pós graduados.

Os mestres exercem suas atividades nos seguintes regimes de trabalho: $2 \mathrm{em}$ regime de 20 horas, $1 \mathrm{em}$ regime de 40 horas e $2 \mathrm{em}$ regime de dedicação exclusiva. Dos especialistas 6 encontram-se no regime de 20 horas, 7 exercem atividades em regime de 40 horas e 5 encontram-se em dedicação esclusiva.

Com relação à ocupação do pós graduado verificamos que 16 docentes, correspondendo a $72,8 \%$ exercem exclusivamente a docência. $O$ restante está assim distriburdo: $18,2 \%$, ou seja, 4 docentes acumulam a docência com atividades administrativas (chefia de departamento, coordenadoria de colegiado, orientação discente), um docente encontra-se cursando mestrado e outro colocado à disposição de outra Instituição.

\section{4 - A Situaçăo Atual da Pós Graduaçăo em Enfermagem na Fundaçăo Edu- cacional do Pará.}

A Fundação Educacional do Estado do Pará (FEP) mantém a Escola de Enfermagem Magalhães Barata. Funciona desde 1944 e of erece habilitaçōes em Enfermagem Médico Cirúrgica, Enfermagem Materno Infantil e Enfermagem de Saúde Pública.

Em 1974 foi realizado o Curso de Enfermagem do Trabalho em convênio com a Fundacentro, com carga horária de $\mathbf{4 7 5}$ horas, tendo sido of ertadas $\mathbf{4 0}$ vagas. Em 1981 foi realizado o 2. Curso de Especialização - Tendências Atuais da Enfermagem, com carga horária de 585 horas e em convênio com a FEP/SUDAM/FADESP.

A política de capacitação de recursos humanos é feita através do encaminhamento de docentes a outras IES.

Em termos de perspectivas há a necessidade da realização de cursos de "lato sensu" como objetivo da melhoria do ensino. Encontra-se programado o Curso Materno Infantil o qual depende de recursos financeiros para a sua realização.

A Escola de Enfermagem Magalhães Barata possui 37 docentes enfermeiros dos quais 32 são pós graduados, sendo 5 mestres e 27 especialistas. Do total de 37 docentes 3 encontram-se com suspensão de contrato.

\section{5 - Situaçăo Atual da Pós Graduação em Enfermagem na Fundaçåo Fede- ral do Maranhăo.}

O Curso de Enfermagem da Universidade Federal do Maranhão foi criado em 1948 propondo-se a formar enfermeiros generalistas. Não of erece habilitações.

Possui um Departamento com 27 docentes enfermeiros contando ainda com a colaboração de docentes de outros departamentos que auxiliam na formação do enfermeiro.

Em 1982 foi realizado um Curso de Especialização em Enfermagem Obstétrica, com carga horária de 780 horas. Foram of ertadas 20 vagas tendo concluído apenas 15. Ainda nesse ano foi of ertado novo curso de especialização denominado Enfermagem Médico Cirúrgica, com carga horária de 570 horas. Foram of ertadas 15 vagas a docentes, enfermeiros e assistentes, sendo que apenas 10 concluiram o curso.

No ano de 1984 foi realizado outro curso de especialização em Enfermagem Médico Cirúrgica destinado à mesma clientela do anterior - enfermeiro, docentes e assistentes. Com uma carga horária de 585 horas, o curso of ereceu 15 vagas tendo concluído apenas 10 enfermeiros.

No período de 1984/1985 foi realizado o Curso de Enfermagem Psiquiátrica, com 735 horas. As vagas of erecidas foram em número de 20 , tendo conclurdo apenas 11 candidatos. Os cursos "lato sensu" ofertados pelo Departamento de Enfermagem são realizados durante o recesso escolar.

Dentro da política de capacitação de recursos humanos, os docentes são encaminhados a outros centros. Em termos de perspectivas sente o Departamento de Enfermagem a necessidade de ampliação de cursos de pós graduação, em caráter permånente.

Em relação à pesquisa há necessidade de um lastro científico, representado por números significativos de pesquisas e publicaçōes que possam vir a prestar serviços à comunidade.

O Departamento de Enfermagem utiliza em seu quadro de pessoal o professor substituto.

A Universidade Federal do Maranhão possui, no Departamento de Enfermagem 4 mestres e 21 espe- 
cialistas de um total de 27 docentes enfermeiros o que corresponde a $92,5 \%$ de pos-graduado.

A Escola de Enfermagem utiliza 3 docentes em atividade de pesquisa.

\section{6 - Situação Atual da Pós Graduação em Enfermagem na Escola de Enfer- magem de Manaus.}

A Escola de Enfermagem de Manaus - EEM iniciou seu funcionamento no ano de 1950. É uma unidade de ensino superior isolada mantida pelo Ministério da Saúde, através da Fundação Serviço Especial de Saúde Pública - FSESP. Possui 03 Departamentos em sua estruturação: Departamento de Ciências Básicas, Departamento de Enfermagem Médico Cirúrgica e Departamento de Enfermagem Materno Infantil e Saúde Pública.

Oferece habilitaçōes em Enfermagem Médico-Cirúrgica, Enfermagem de Saúde Pública e Enfermagem Materno Infantil, além de manter o Curso Intensivo de Auxiliar de Enfermagem.

A política de capacitação docente é direcionada aos grandes centros de pós graduação. Seu quadro funcional é de 30 docentes enfermeiros privativos em tempo integral e dedicação exclusiva e 24 docentes colaboradores, não enfermeiros.

Não é of erecido regularmente curso de pós graduação na referida escola.

Em 1981 foi realizado o 1. Curso de Pós Gra-* duação a nível de especialzação em convênio EEM/FSESP/PICD/UFRJ denominado de Metodologia do Ensino, da Pesquisa e da Assistência de Enfermagem, com carga horária de 540 horas, com 08 vagas destinadas a clientela enfermeiro assistentes e docentes. Posteriormente em 1985 no $2^{\circ}$. semestre foi fealizado o $2^{\circ}$. Curso de Especialização com a mesma denominação, carga horária, clientela, alterada o número de vagas para 20.

Dos 30 docentes enfermeiros privativos 2 possuem título de Doutor, 7 são mestres e 16 possuem cursos de especialização, nota-se ainda que a Escola de Enfermagem de Manaus encontra-se com 2 docentes enfermeiros realizando curso de mestrado.

A quase totalidade dos docentes enfermeiros, em número de 25 , exercem apenas a docência. Não se registra a atividade de pesquisa ou extensão, naquela escola.

Podemos constatar, portanto, que é o seguinte o quadro de pós-graduados em enfermagem na Região Norte do Brasil:

\begin{tabular}{c|c|c|c|c}
\hline \multicolumn{1}{c|}{ I.E.S. } & Doutor/Livre Docente & Mestre & Especialistas & Total \\
\hline Universidade Federal do Acre & - & 4 & 18 & 22 \\
Universidade F. de M. Grosso & - & 6 & 39 & 45 \\
Universidade Federal do Pará & - & 5 & 18 & 23 \\
E.E. Magalhães Barata (Pará) & - & 5 & 27 & 32 \\
Universidade F. do Maranhão & - & 4 & 21 & 25 \\
E. de Enfermagem de Manaus & 2 & 7 & 16 & 25 \\
\hline TOTAL & 2 & 31 & 149 & 182 \\
\hline
\end{tabular}

Fonte: Pesquisa da autora.

Por outro lado podemos ainda afirmar que embora a Região Norte conte com 182 docentes enfermeiros pós graduados a grande maioria realizou seus cursos em IES de outras regiōes. Dos cursos de pós graduação ofertados pelas Instituiçōes de Enfermagem da Amazônia Legal, nenhum deles é de mestrado. Em nível de aperfeiçoamento somente um curso foi ministrado, pela Universidade Federal do Acre. Em nível de especialização foram ministrados apenas 9 cursos, no perfodo de 1981 a 1986, pelas Universidades Federais do Acre, Maranhão, Mato Grosso, Pará e pela Escola de Enfermagem de Manaus. Dos 9 cursos ministrados a Universidade Federal do Maranhão ofertou 4, a Escola de Enfermagem de Manaus of ereceu 2 enquanto que as Universidades Federais do Acre, Matto Grosso e Pará ministraram apenas 1 , cada.
Os docentes que realizaram curso de mestrado apresentaram em tempo hábil sua dissertação. Da análise dos assuntos tratados nas dissertaçōes, verificamos que na sua quase totalidade foram pesquisas realizadas fora de uma realidade regional. Levando em conta que a pesquisa era desenvolvida no local do curso realizado, freqüentemente as pesquisas retratavam realidades daquelas regiōes, em detrimento do que pusse ser pesquisado e relatado em relação à Instituição de origem.

\section{CONCLUSÃO}

O desenvolvimento do corpo docente assim como o aperfeiçoamento do ensino e a realização da investigação cientifica encontram sérias dificuldades nas instituições de enfermagem da Amazónia Legal, seja 
por falta de recursos financeiros, seja pela dificuldade de deslocamento de docentes para outras IES, em tempo mais prolongado, qual seja o da realização de um Curso de Mestrado.

Verificamos que não foi atingido o nível minimo almejado para alcançar um quantitativo de doutores e mestres em enfermagem, em relação ao total de docentes.

Por outro lado, se apenas 2 doutores estão relacionados, verificamos que a Universidade Federal do Acre encaminha seu primeiro docente para realizar aquele curso.

Os cursos de especialização não são of ertados, em nenhuma das instituições, de forma permanente.

Carecem, portanto, as instituições de enfermagem da Amazônia Legal, de maior apoio não apenas de recursos financeiros como de pessoal pós graduado para ministração de cursos, não apenas em nível de "lato sensu" como "stricto sensu", em caráter permanente.

As disposições estruturais da sociedade responsabiliza-nos a admitir que não se dese ja apenas o preparo do profissional de enfermagem de alto nível para a assistência da enfermagem mas, sobretudo a qualificação de docentes para que possam formar melhores enfermeiros, a nivel de graduação.

Pela constatação do número de mestres existentes sugerimos a realização, em uma das instituições de enfermagem da Amazônia Legal, de uni curso de mestrado em enfermagem, apresentado em modulos, a fim de facilitar a qualificação de maior número de docentes permitindo inclusive que grande número de especialistas possam titular-se em mestre, o que não vem ocorrendo não apenas pelas dificuldades de locomoção para outras IES, como também por problemas familiares.

Considerando-se, ainda que as Instituições de Enfermagem da Amazônia Legal, contam com 2 doutores, além de número suficiente de mestres podem eles serem convocados para ministrar curso, o qual deveria ser of ertado para todos os interessados, preferencialmente docentes, das Instituições de Enfermagem da Amazôna Legal o que traria benefícios não apenas para a docência, mais também para o desenvolvimento de pesquisa científica.

\section{REFERÊNCIAS BIBLIOGRÁFICAS}

BASSALO, C.C. et alii. Pesquisa e pós graduação em debate.Belém, Universidade Federal do Pará, 1984. mimeogr.

2 FUNDAÇÃO UNIVERSIDADE FEDERAL DE MATO GROSSO. A pós graduaçāo na UFMT: Documento básico. Manaus, 1986, $11 \mathrm{p}$.

3 GONÇALVES, L.H.T. Situação atual do curso de mestrado em ciências da enfermagem, opção saúde do adulto, do departamento de enfermagem do centro de Ciências da Saúde da Universidade Federal de Santa Catarina.

In: SEMINÁRIO NACIONAL SOBRE ENSINO DE PÓS GRADUAÇÃO E PESQUISA EM ENFERMAGEM. Brasília, 1982. Avaliação e perspectivas. Brasilia, CNPQ/ABEN, 1982, p. 19-24.

4 INSTITUTO NACIONAL DE PESQUISA DA AMAZÔNIA. Cursos de pós graduação do convênio INPA/FUA. Docionento básico. Belém, INPA/FUA, 1986, 11 p.

5 MACHADO, E. A CAPES e a pós graduação na Regiðo Norte, documento básico. Belém, 1986, 7 p. mimeogr.

6 PELÁ, N. T. R. et alii. Situação atual do cursos e produção científica da pós graduação da Escola de Enfermagem de Ribeirão Preto. In: SEMINÁRIO NACIONAL SOBRE ENSINO DE PÓS GRADUAÇÃO E PESQUISA EM ENFERMAGEM. Brasília, 1982. Avaliação e perspectivas. Brasilia, CNPQ/ABEN, 1982, p. 8-18.

7 RHODUS, C. C. Pós Graduaçào em enfermagem: Um estudo. Rio de Janeiro, UFRJ, Escola de Enfermagem Ana Neri, 1977. tese Liv. Doc. 69p.

8 UNIVERSIDADE FEDERAL DO ACRE. PróReitoria de Pesquisa e Pós Graduação. Pós Graduação na Amazônia: Uma necessidade. Rio Branco, 1986, 15 p. mimeogr.

9 UNIVERSIDADE FEDERAL DO PARÁ. Pró Reitoria de Pesquisa e Pós Graduação. Projeto Norte de Pós Graduaçðo: Documento básico. Belém, 1986, 25 p. mimeogr. 